\title{
Pericytes for Therapeutic Approaches to Ischemic Stroke
}

\author{
Lu Cao ${ }^{1}$, Yanbo Zhou ${ }^{1}$, Mengguang Chen ${ }^{1}, \mathrm{Li} \mathrm{Li}^{2 *}$ and Wei Zhang ${ }^{1 *}$ \\ ${ }_{1}^{1}$ Department of Anesthesiology, Pain and Perioperative Medicine, The First Affiliated Hospital of Zhengzhou University, \\ Zhengzhou, China, ${ }^{2}$ Department of Anesthesiology, Beijing Friendship Hospital, Capital Medical University, Beijing, China
}

OPEN ACCESS

Edited by:

Yujie Chen,

Army Medical University, China

Reviewed by:

Anusha Mishra,

Oregon Health and Science

University, United States

Qiang Li,

University of Texas Medical Branch,

United States

Berislav Zlokovic,

University of Southern California,

United States

${ }^{*}$ Correspondence:

$\mathrm{Li} \mathrm{Li}$

li_anethesia@163.com; lili2009kaoyan@163.com

Wei Zhang

chalou666666@126.com

Specialty section:

This article was submitted to Neurogenesis,

a section of the journal

Frontiers in Neuroscience

Received: 14 November 2020 Accepted: 11 May 2021

Published: 18 June 2021

Citation:

Cao L, Zhou Y, Chen M, Li L and Zhang W (2021) Pericytes for Therapeutic Approaches to Ischemic Stroke.

Front. Neurosci. 15:629297. doi: 10.3389/fnins.2021.629297
Pericytes are perivascular multipotent cells located on capillaries. Although pericytes are discovered in the nineteenth century, recent studies have found that pericytes play an important role in maintaining the blood-brain barrier (BBB) and regulating the neurovascular system. In the neurovascular unit, pericytes perform their functions by coordinating the crosstalk between endothelial, glial, and neuronal cells. Dysfunction of pericytes can lead to a variety of diseases, including stroke and other neurological disorders. Recent studies have suggested that pericytes can serve as a therapeutic target in ischemic stroke. In this review, we first summarize the biology and functions of pericytes in the central nervous system. Then, we focus on the role of dysfunctional pericytes in the pathogenesis of ischemic stroke. Finally, we discuss new therapies for ischemic stroke based on targeting pericytes.

Keywords: pericytes, ischemic stroke, cerebral blood flow, blood-brain barrier, therapeutic strategy

\section{INTRODUCTION}

Pericytes are perivascular multipotent cells located on capillaries. The perivascular cell was first discovered by Rouget in 1873, so it was named "Rouget's cell" (Rouget, 1873). Furthermore, according to the morphology and location of Rouget's cell, interacting with the underlying endothelial cells (ECs) that shared the basement membrane, Zimmermann renamed it "pericyte" in 1923 (Zimmermann, 1923). Subsequently, more studies discovered the origin and functions of pericytes. Pericytes in cerebral vessels develop from different layers of the germ. Faal et al. (2019) pointed out that pericytes of the central nervous system (CNS) were mainly derived from mesenchymal stem cells of the mesoderm. Some studies also discovered that pericytes of the forebrain originated from nerve crest cells, while pericytes of the midbrain, brain stem, and spinal cord originated from mesoderm stem cells (Etchevers et al., 2001; Korn et al., 2002; Kurz, 2009). There are pieces of evidence suggesting that pericytes in the brain can exert a large variety of functions (Bell et al., 2010; Armulik et al., 2011), including regulating cerebral blood flow (CBF), maintaining BBB integrity, regulating angiogenesis and inflammation, and acting as stem cells and progenitor cells (Gautam and Yao, 2018).

Ischemic stroke is a cerebrovascular disease due to the blockage of blood vessels and decreased blood supply in relevant brain regions (Li et al., 2014). When ischemic stroke occurs, a series of molecular and cellular events occur, ultimately leading to disruption of CBF, destruction of the blood-brain barrier (BBB), inflammation, glial cell activation, vascular malformation, and neuronal death (Moskowitz et al., 2010; Terasaki et al., 2014). Recent studies show that pericytes influence ischemic stroke pathology and contribute to progression and recovery.

In this review, we focus on recent studies on the biology and functions of pericytes during ischemic stroke. Specifically, we discuss the role of pericytes in CBF, BBB integrity, angiogenesis, 
immune response, and scar formation and fibrosis (Table 1). Finally, we address recent findings on treatment strategies for ischemic stroke by targeting pericytes.

\section{Pericytes and Cerebral Blood Flow in Ischemic Stroke}

The question of whether pericytes can regulate $\mathrm{CBF}$ is still debatable. Pericytes have been demonstrated to regulate blood flow to the brain. For instance, actin and myosin-like filaments have been found in rat pericerebral cells (Le Beux and Willemot, 1978). Moreover, many kinds of contractile proteins, such as smooth muscle actin (SMA), have been identified in the culture of pericerebral cells, indicating that pericytes can synthesize the protein (Sieczkiewicz and Herman, 2003; DoreDuffy and LaManna, 2007). Consistent with these findings, pericytes cultured in vitro have shown contraction due to intracellular $\mathrm{Ca}^{2+}$ (Kamouchi et al., 2004). Some studies reported that glutamate-induced capillary pericytes are mediated by prostaglandin E2 and nitric oxide, and vasodilation occurs before the arteriole dilation caused by electrical stimulation to increase blood flow (Hall et al., 2014; Kisler et al., 2017). Besides, Kisler et al. used pericyte-specific Cre mouse model with Cre-dependent human diphtheria toxin (DT) receptor (Kisler et al., 2020). DT led to rapid progressive loss of pericyte coverage of cortical capillaries; however, endothelial response, microvascular density, and neuron-evoked membrane potential responses remained. This study suggested that neurovascular uncoupling is driven by pericyte loss, not other vascular or neuronal dysfunction. The result supported the role of pericytes in $\mathrm{CBF}$ regulation (Kisler et al., 2020). Together, these results suggest that pericytes take an active part in regulating $\mathrm{CBF}$ under physiological conditions.

Pericyte contraction can be observed under pathological conditions as well, such as stroke. Research showed that in the process of stroke, pericytes could entrap red blood cells in the capillary contraction part, which obstruct the microcirculation (Yemisci et al., 2009). Similarly, Hall et al. (2014) reported that pericytes contracted capillaries and died quickly after ischemia, and pericyte death led to permanent capillary contraction; furthermore, the decreased time of CBF was prolonged even when arterial blood flow was restored. These pathological changes in pericytes after stroke were duplicated with the iCelligence electrical impedance system. Neuhaus et al. (2017) confirmed that chemical ischemia induced long-term and irreversible contraction of pericytes before death in vitro. These results suggest that the contraction and death of pericytes are involved in the pathogenesis of stroke by regulating $\mathrm{CBF}$. Further studies have shown that the cause of pericyte death is partially mediated by glutamate, while free radical scavenging does not reduce pericyte death (Hall et al., 2014). Compared to this report, inhibition of nitrate-mediated oxidative stress has been demonstrated to reduce pericyte contraction induced by ischemia reperfusion and have a positive effect on tissue survival (Yemisci et al., 2009). In addition, in the rat model of focal cerebral ischemia, eddaravan, a free radical scavenger, can reduce infarct size by preventing pericyte contraction while promoting pericyte proliferation (Deguchi et al., 2014). Recently,
Nelson et al. (2020) examined pericyte contractility using a new optogenetic model by inducing pericyte-specific CreER mouse line and ChR2 mouse and monitored and confirmed pericyte contractility in vivo and regulated capillary blood flow in the brain of aging mice under a two-photon microscope. Besides, Hartmann et al. (2021) also found that brain capillary pericyte optogenetic stimulation decreased lumen diameter and blood flow, but with slower kinetics than similar stimulation of mural cells on upstream pial and precapillary arterioles.

On the contrary, there are some researches indicating that pericytes cannot contract and regulate $\mathrm{CBF}$ as well. Using a variety of transgenic mice and two-photon microscopy, Hill et al. (2015) did not detect SMA expression in mice and human pericytes. They also showed that changes in blood vessel diameter and blood flow caused by optogenetic whisker stimulation and cortical spreading depolarization occurred in the microvessels covered by smooth muscle cell (SMC) but not in the capillaries covered by pericytes (Hill et al., 2015). In addition, by using a short-term middle cerebral artery occlusion (MCAO) model, it has been found that SMC contraction rather than pericyte contraction resulted in hypoperfusion and thus distal microvascular occlusion. Fernández-Klett et al. (2010) also found that the increase in CBF caused by neural activity can be explained by precapillary and penetrating arterioles, rather than pericyte in capillaries. These studies suggest that SMC, rather than pericyte, contributed to the regulation of blood flow under both physiological and pathological conditions. The difference is probably due to the similar structural and functional properties of pericytes and precapillary SMC (Hartmann et al., 2015), making it difficult to distinguish them completely. Some groups showed synaptic activity generated a synchronous $\mathrm{Ca} 2^{+}$drop in pericytes and SMCs resulting in telangiectasia starting mainly from primary or secondary capillaries and then spreading along arterioles and downstream capillaries, which may be the location of pericytes in the proximal capillaries as the main regulator of CBF (Cai et al., 2018; Khennouf et al., 2018; Rungta et al., 2018). However, the main divergence of the two opinions is the definition of pericyte, since Hill et al. (2015) and Fernández-Klett et al. (2010) defined SMA-expression capillary cell as SMC, while Hartmann et al., 2015 defined it as pericyte. The findings of Hill et al. (2015) and Fernández-Klett et al. (2010) are identical to other findings once definition differences are taken into account. In summary, most of the studies show that pericytes with SMA expression can regulate $\mathrm{CBF}$.

\section{Pericytes and the Blood-Brain Barrier in Ischemic Stroke}

The BBB is a dynamic barrier between blood and brain tissue that acts as a selective barrier to substances. Pericytes play an important role in the formation and maintenance of the BBB. In the CNS, a simplified neurovascular unit is composed of vascular cells (pericytes, vascular SMCs, ECs), glial cells (astrocytes, microglia, oligodendrocytes), and neurons (Figure 1). Pericytes are important components of the neurovascular unit and BBB. Daneman et al. (2010) have found that pericyte recruitment occurred 1 week before astrocyte recruitment during the 
TABLE 1 | Functions of pericytes in ischemic stroke.

\begin{tabular}{|c|c|c|}
\hline Functions & Roles & References \\
\hline Regulating cerebral blood flow & Capillary contraction & $\begin{array}{l}\text { Yemisci et al., 2009; Hall et al., 2014; Neuhaus et al., 2017; } \\
\text { Kisler et al., } 2020\end{array}$ \\
\hline Formation and maintenance of BBB & Regulation of vascular permeability Modulation of BBB integrity & Dohgu et al., 2005; Bai et al., 2015; Daneman and Prat, 2015 \\
\hline Angiogenic property & Blood vessel stabilization Revascularization & Dore-Duffy and LaManna, 2007; Stenzel et al., 2009 \\
\hline Immunological characteristics & Releasing anti-inflammatory cytokines/chemokines & $\begin{array}{l}\text { Dohgu and Banks, 2013; Sakuma et al., 2016; Duan et al., } \\
2018\end{array}$ \\
\hline Scar formation and fibrosis & Stem cell potential expressing PDGFR $\beta$ Neuroprotection & Makihara et al., 2015; Sakuma et al., 2016; Ozen et al., 2018 \\
\hline
\end{tabular}

BBB, blood-brain barrier; PDGFRß, platelet-derived growth factor receptor-beta.

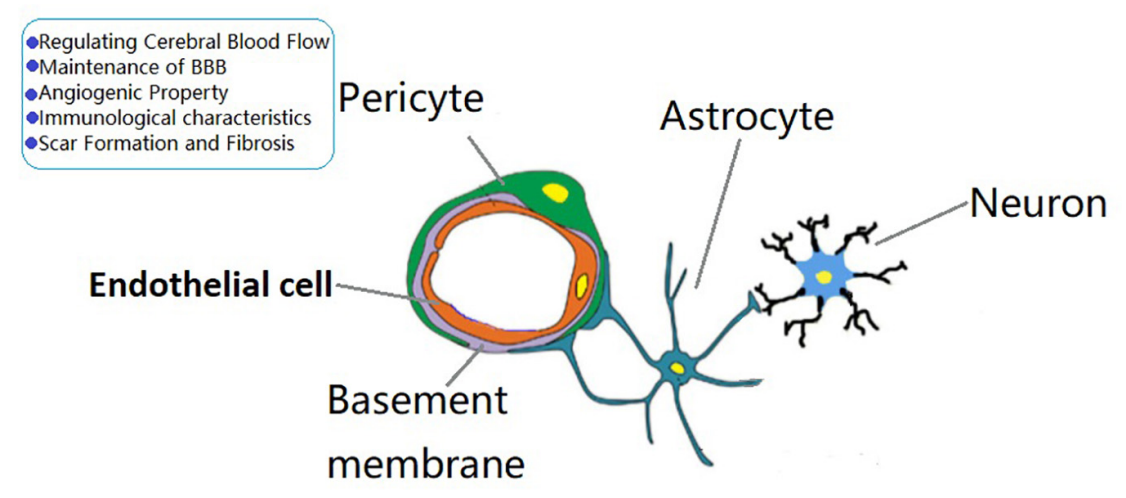

FIGURE 1 | The role of brain pericytes at the neurovascular unit (NVU). A simplified NVU is composed of vascular cells [pericytes, vascular smooth muscle cells, endothelial cells (ECs)], glial cells (astrocytes, microglia, oligodendrocytes), and neurons. Pericytes have the functions of regulating cerebral blood flow, maintenance of blood-brain barrier permeability, angiogenic characteristcs, immune responses, and scar formation and fibrosis.

formation of BBB. They also found that the coverage of pericyte played a crucial role in vascular permeability, and pericytes regulated $\mathrm{BBB}$ at the endothelial connectivity level (Daneman and Prat, 2015). Bell et al. (2012) found that apolipoprotein E maintained cerebrovascular integrity necessary for normal neuronal function by regulating the cyclophilin A-nuclearfactor- $\kappa \mathrm{B}$-matrix metalloproteinase pathway in pericytes in an isoform-specific manner. Al Ahmad et al. (2009) found that the increased integrity of endothelial barrier was related to pericytes and astrocytes, and pericytes protected the endothelial barrier better than astrocytes after thymic ischemia.

Several studies have shown that the loss of pericyte coverage led to destruction of $\mathrm{BBB}$ and endothelial barrier functions. Pericytes maintain $\mathrm{BBB}$ function by releasing angiopoetin1 (Ang-1) and transforming growth factor-beta1 (TGF- $\beta 1$ ). Increased occludin expression in ECs is associated with pericyte release of Ang-1. However, low levels of occludin cause the breakdown of tight junction (TJ) and blood vessel permeability (Hori et al., 2004). Dohgu et al. (2005) discovered that pericyte-derived TGF- $\beta 1$ had the ability to directly influence $\mathrm{BBB}$ function. TGF- $\beta 1$ activates mitogen-activated protein kinase (MAPK) signaling to increase the expression of $\mathrm{TJ}$ protein and $\mathrm{P}$-glycoprotein (P-gp) expressed in EC to improve BBB function. However, Shen et al. (2011) showed that TGF- $\beta 1$ promoted tyrosine phosphorylation of vascular endothelial-cadherin and claudin-5 in ECs and enhanced paracellular permeability. The role of pericyte-derived TGF- $\beta 1$ in ischemic stroke needs further studies. Besides, Zlokovic (2013) showed that loss of pericytes disrupted cerebrovascular integrity and led to microvascular reductions amplifying vascular damage.

Although pericyte dysfunction is not directly associated with ischemic stroke, pericytes may be indirectly involved in ischemic pathogenesis by affecting vascular integrity. There is evidence that pericytes regulate $\mathrm{BBB}$ integrity through vascular endothelial growth factor (VEGF), thereby regulating ischemic injury. Sodium cyanide $(\mathrm{NaCN})$ was used in an in vitro ischemia model, and it has been found to significantly increase the expression of VEGF in brain pericytes, while the conditioned medium from $\mathrm{NaCN}$-treated pericytes destroyed vascular integrity in the in vitro BBB model (Bai et al., 2015). Meanwhile, it has been shown that VEGF mediated the increased permeability of $\mathrm{BBB}$ in ischemic brain (Zhang et al., 2000). In contrast, prolonged exposure to VEGF has been shown to improve the integrity of the BBB after ischemia (Zechariah et al., 2013). This difference may be due to differences in dose, treatment measures, and timing. Furthermore, pericytes influence BBB integrity through reactive oxygen species (ROS), thereby affecting the ischemic process. Studies have shown that nicotinamide adenine dinucleotide phosphate (NADPH) oxidase 4 (NOX4) is highly upregulated in the peri-infarct area in the MCAO model. NOX4 has been shown to be primarily derived from pericytes, and the expression of NOX4 in pericyte was significantly increased in peri-infarct areas after MCAO. Further studies 
showed that the overexpression of NOX4 in pericyte caused BBB destruction through upregulation of metalloproteinase 9, especially emphasizing the significant role of ROS in BBB integrity (Nishimura et al., 2016).

\section{Angiogenic Property of Pericytes in Ischemic Stroke}

Pericytes are closely related to the formation and stabilization of angiogenesis. The recruited pericytes enter new blood vessels to promote vascular maturation by releasing paracrine factors such as platelet-derived growth factor receptor-beta (PDGFR $\beta$ ) (Dore-Duffy and LaManna, 2007). It has been reported that pericytes could stabilize the formation of capillary-like structures (Ramsauer et al., 2002). Persidsky et al. (2006) showed that pericytes were involved in the regulation of EC migration, proliferation, and differentiation. Furthermore, pericytes were reported to mediate the formation of new blood vessels through certain signaling molecules such as PDGFR $\beta$, TGF- $\beta$, VEGF, and Ang-1 (Dore-Duffy and LaManna, 2007). Pericytes release VEGF to promote EC maturation (Hagedorn et al., 2004). Pericyte-derived TGF- $\beta$ induces perivascular mesenchymal cells to differentiate into pericyte and SMCs (Sinha et al., 2004). Pericyte-released Ang-1 binds to Tie-2 in ECs, thereby promoting heparin binding to epidermal growth factor-like growth factor (HB-EGF) expression, which plays a key role in vascular stability (Stenzel et al., 2009).

Stroke is a cerebral blood circulation disease caused by stenosis, occlusion, or rupture of intracerebral arteries. Rebuilding blood flow to the brain in damaged areas aids in stroke recovery. Based on the important role of pericytes in angiogenesis, it is supposed that pericytes may contribute to stroke recovery by regulating angiogenesis. Recombinant human VEGF in the MCAO model has been shown to promote capillary growth and pericyte coverage, promote cerebral blood circulation, and thus reduce cerebral infarction area (Zechariah et al., 2013). In keeping with these observations, VEGF receptor inhibition accelerates cell death, inhibits EC proliferation, and exacerbates injury in neonatal stroke models (Shimotake et al., 2010). Based on these studies, it is speculated that pericytes have a positive effect in ischemic stroke by promoting angiogenesis.

It is important to point that angiogenesis may also have a negative effect on ischemic stroke. For example, elevated VEGF expression during ischemia was related to uncoupling of EC connectivity and increased vascular permeability and edema (Weis and Cheresh, 2005). In keeping with this finding, VEGF antagonism inhibited the formation of edema and tissue damage to some extent after MCAO in mouse brain (Nicholas van Bruggen et al., 1999). Furthermore, enhanced angiogenesis, including promoted EC activation and retinal hypervascularization, was observed in Notch1 ${ }^{ \pm}$Notch3 ${ }^{-/-}$ mice, a model of cerebral autosomal dominant arteriopathy with subcortical infarcts and leukoencephalopathy (CADASIL) (Kofler et al., 2015). In addition, an increased incidence of ischemic stroke was related to type 1 and type 2 diabetes (Janghorbani et al., 2007). Angiogenesis in diabetes is persistent and uncontrolled (Cheng and Ma, 2015). In a word, pericyte angiogenic properties may play a dual role in ischemic stroke due to differences in the animal model, timing, and types of injury.

\section{Immunological Characteristics of Pericytes in Ischemic Stroke}

Pericytes take part in CNS defense by exhibiting non-specific and specific immune responses. A number of studies have reported that pericytes responded to pro-inflammatory signals. For example, chemokines and cytokines, including granulocyte colony-stimulating factor (G-CSF), interleukin (IL)-1 $\alpha$, IL-6, and nitric oxide, are constitutively produced in brain pericytes of mice in normal conditions. In lipopolysaccharide (LPS)-induced inflammation, changes in expression levels of these chemokines and cytokines were accompanied by the induction of many new factors such as IL-5 and regulated upon activation normal T cell expressed and secreted factor (RANTES) (Kovac et al., 2011; Dohgu and Banks, 2013). With the increase of age and injury, pericytes with high expression of lysosomal acid phosphatase have phagocytosis. Pericytes may absorb substances in the blood or brain parenchyma through various endocytoses. When the $\mathrm{BBB}$ is broken down, pericytes can engulf the red blood cells (Castejon, 1984). Meanwhile, cultured rat pericytes expressed macrophage markers ED-2 and CD11b and were phagocytic with fluorescent dye-conjugated polystyrene beads and antibodycoated yeast polysaccharide, suggesting Fc receptor-independent and -dependent phagocytic activity (Balabanov et al., 1996). In summary, pericytes may have the immune cell-like characteristics and the ability to modulate immune responses.

Stroke is also a nervous system disease associated with the local inflammatory reactions and an immune response in the brain. It is supposed that pericytes may respond to CNS damage, including ischemic stroke, by transforming into microglia/macrophage-like cells (Boya, 1976). More recently, some studies have suggested that pericytes may acquire the microglia-like properties after ischemic stroke. First, it has been reported that the cells that express the regulator of G-protein signaling 5 (RGS5) after cerebral ischemia injury were mainly pericytes (Bondjers et al., 2003; Cho et al., 2003; Berger et al., 2005), which is associated with the proliferation and production of $\mathrm{CD} 11 \mathrm{~b}+$ and galactosin $3+$ microglia cells (Ozen et al., 2018). Secondly, pericytes of the human brain acquire stemness characteristics and differentiate into a variety of lineages including microglia cells under oxygen and glucose deprivation simulating ischemic injury in vitro (Nakagomi et al., 2015). Meanwhile, pericyte stemness characteristics were detected in the ischemic regions of the mice, indicating that pericytes had multipotency in ischemic induction (Nakagomi et al., 2015; Gouveia et al., 2017). In addition, Iba1 + microglia cells express PDGFR $\beta$ in ischemic encephalopathy, showing that some microglia may come from post-ischemic pluripotent pericytes (Sakuma et al., 2016). Additionally, PDGFR $\beta+$ pericytes isolated from ischemic differentiated into microglia-like cells and acquired phagocytic activity (Sakuma et al., 2016). Recently, it has been reported that within $2 \mathrm{~h}$ of systemic inflammation, PDGFR $\beta$ mural cells of blood vessels rapidly secreted chemokine CC-chemokine ligand 2 (CCL2), which in turn increased 
total neuronal excitability by promoting excitatory synaptic transmission in glutamatergic neurons of multiple brain regions (Duan et al., 2018). The study demonstrated in vivo that PDGFR $\beta$ cells functioned as initial sensors of external insults by secreting CCL2, which relayed the signal to the CNS. Through their gateway position in the brain, PDGFR $\beta$ cells are ideally positioned to respond rapidly to environmental changes and to coordinate responses. In summary, these studies show that pericytes can differentiate into microglia-like cells and perform related functions in ischemic conditions.

\section{Pericytes and Scar Formation and Fibrosis in Ischemic Stroke}

After injury of the CNS, glial cells are activated and form glial scar at the injury region by deposition of chondroitin sulfate proteoglycans, including neuroproteoglycans and phosphatase proteoglycans (Fawcett and Asher, 1999; McKeon et al., 1999; Silver and Miller, 2004; Li et al., 2013; Cregg et al., 2014). The function of scar tissue can keep toxic substances from spreading throughout the CNS (Fitch and Silver, 2008; Kawano et al., 2012). However, excessive or prolonged scar formation can inhibit axonal regeneration and hinder the recovery process, leading to fibrosis (Höke and Silver, 1996; Asher et al., 2001; Fitch and Silver, 2008). Besides, it is reported that astrocyte scar formation contributes to rather than prevents CNS axon regeneration (Anderson et al., 2016). The study showed that preventing or attenuating scar-forming astrocytes or ablating chronic astrocytic scars all failed to generate spontaneous regrowth of serotonergic axons in spinal cord injury (SCI) lesions. Sustained local delivery via hydrogel depots of required axonspecific growth factors not present in SCI lesions stimulated robust, laminin-dependent sensory axon regrowth past scarforming astrocytes and inhibitory molecules in SCI lesions. By contrast, preventing astrocytic scar formation significantly reduced this stimulated axon regrowth.

It has been suggested that pericytes promoted scar formation and organ fibrosis. Pericytes are categorized into type I (Nestin-GFP ${ }^{-} / \mathrm{NG}^{-}$-DsRed ${ }^{+}$) and type II (Nestin-GFP ${ }^{+} / \mathrm{NG} 2-^{-}$ DsRed $^{+}$), producing adipocyte/fibroblast and nerve/myogen cells, respectively (Birbrair et al., 2013a,b). It has been reported that pericytes of type I gathered and contributed to scar formation in multiple organs (including brain, spinal cord, myocardium, and kidneys) after injury (Birbrair et al., 2014). It has been reported that in ischemic injury, $\operatorname{PDGFR} \beta^{+}$pericytes induced the fibrosis response in the kidney and CNS (Chen et al., 2011; Makihara et al., 2015). Compared to the control group, $\mathrm{PDGFR}^{ \pm}$ mice showed reduced fibrosis, reduced fibronectin deposition, and increased infarct size in the ischemic area, showing that PDGFR $\beta$ signaling-induced fibronectin production was essential for the repair process after ischemic stroke (Makihara et al., 2015). This discovery agrees with the neuroprotective effect of fibronectin in CNS injury (Tom et al., 2004; Yanqing et al., 2006).

\section{Pericytes for Therapeutic Approaches to Ischemic Stroke}

As discussed above, pericytes are involved in maintaining normal cerebrovascular function and play an important role in the pathological process of ischemic stroke. Thus, targeting pericytes may be an effective therapeutic method for ischemic stroke (Dalkara, 2019).

RGS5 protein that regulates vascular development was identified as a biomarker of pericytes (Bondjers et al., 2003). Ozen et al. (2018) found that the number of pericytes increased and the damage to the BBB decreased significantly after the RGS5 gene was knocked out in the model of permanent midbrain occlusion. In addition, loss of RGS5 in pericytes maintained aquaporin-4 (AQP4) expression in astrocytes and the integrity of TJs in ECs, reduced cerebral hypoxia, alleviated vascular leakage, and protected neurons in ischemic position (Ozen et al., 2018). AQP4 is a water channel protein expressed on astrocytic endfeet and plays an important role in BBB integrity. TJs have an important role in the maintenance of the $\mathrm{BBB}$, and their unique expression in the brain correlates with $\mathrm{BBB}$ permeability. In acute stroke, there is degradation of TJs, resulting in loss of vascular integrity. Thus, targeting RGS5 might be a potential therapeutic strategy for ischemic stroke. Alarcon-Martinez et al. (2019) found that the $\alpha$-SMA-mediated contractility in ischemic stroke and the effect of calcium in regulating contractile response could help in understanding the pericytes related to CBF at single-capillary level in ischemic stroke. Sun M. et al. (2020) found that sentrin/SUMO-specific protease 1 (SENP1) deletion in pericytes exacerbated infarct size and motor dysfunction after cerebral ischemia, though it had no effect on cognitive function. They also found that the deletion of pericyte-specific SENP1 significantly exaggerated neuronal damage after cerebral ischemia in mice. The knockdown of SENP1 in pericytes could activate apoptotic pathways and destroy the integrity of the cell barrier in vitro. These findings suggested that targeting SENP1 in pericytes might be a new therapeutic method for ischemic stroke (Sun M. et al., 2020). Besides, a study found that intracerebroventricular pleiotrophin (PTN) infusions prevented neuronal apoptosis in pericyte-ablated mice from persistent circulatory changes. PTN is a neurotrophic growth factor. The silencing of pericyte-derived PTN rendered neurons vulnerable to ischemic injury. This study demonstrated that pericyte loss was closely related to acute circulatory collapse and loss of PTN support. These findings suggested PTN support might be a new therapeutic method (Nikolakopoulou et al., 2019). Recently, Shibahara et al. (2020) demonstrated that pericyte-mediated fibrosis repair via PDGFR promotes functional recovery by enhancing peri-infarct oligodendrocyte formation and astrocyte proliferation following acute ischemic stroke. Besides, pericytes develop multipotency following experimental ischemia in mice, and these ischemia-induced multipotent stem cells (iSCs) can contribute to neurogenesis. This property of pericytes showed great potential in the treatment of neurovascular diseases (Geranmayeh et al., 2019). Pericytes from induced pluripotent stem cells (iPSC) are also expected to be used in autotransplantation therapy in ischemic stroke as acquiring BBB characteristics and binding to astrocytes, ECs, and neurons (Faal et al., 2019; Stebbins et al., 2019). Pericytes have been shown to acquire properties similar to stem cells and microglia after cerebral ischemia, providing another potential therapeutic strategy for recovery from ischemic stroke (Özen et al., 2014; 
Nakagomi et al., 2015). Recently, Sun J. et al. (2020) generated pericyte-like cells (PCs) from human pluripotent stem cells (hPSCs). They found the cranial neural crest-derived pericytelike cells (hPSC-CNC PCs) expressed typical pericyte markers and showed distinct pericyte properties. Moreover, when transplanted into a mouse model of MCAO with BBB breakdown, hPSC-CNC PCs efficiently improved neurological functional recovery in MCAO mouse model. The study indicated that hPSC-CNC PCs might represent an ideal cell source for the treatment of BBB dysfunction-related disorders and might be a new therapeutic method for ischemic stroke (Sun J. et al., 2020).

Although significant progress has been made in understanding the function of pericytes in the pathogenesis of ischemic stroke, some key issues remain to be studied further. First, although various markers have been used to identify pericytes, there are currently no pericyte-specific markers. It is important to note that the pericytes in most studies contained $\mathrm{PDGFR}^{+}$ cells including both pericytes and SMCs. Secondly, pericytes are a diverse population of cells, and different types of pericytes may play different roles in ischemic stroke. The study in the future should focus on the identification of pericyte-specific and

\section{REFERENCES}

Al Ahmad, A., Gassmann, M., and Ogunshola, O. O. (2009). Maintaining blood-brain barrier integrity: pericytes perform better than astrocytes during prolonged oxygen deprivation. J. Cell Physiol. 218, 612-622. doi: 10.1002/jcp. 21638

Alarcon-Martinez, L., Yilmaz-Ozcan, S., Yemisci, M., Schallek, J., Kılıç, K., Villafranca-Baughman, D., et al. (2019). Retinal ischemia induces $\alpha$-SMAmediated capillary pericyte contraction coincident with perivascular glycogen depletion. Acta Neuropathol. Commun. 7:134.

Anderson, M. A., Burda, J. E., Ren, Y., Ao, Y., O’Shea, T. M., Kawaguchi, R., et al. (2016). Astrocyte scar formation aids central nervous system axon regeneration. Nature 532, 195-200. doi: 10.1038/nature17623

Armulik, A., Genove, G., and Betsholtz, C. (2011). Pericytes: developmental, physiological, and pathological perspectives, problems, and promises. Dev. Cell 21, 193-215. doi: 10.1016/j.devcel.2011.07.001

Asher, R. A., Morgenstern, D. A., Moon, L. D., and Fawcett, J. W. (2001). Chondroitin sulphate proteoglycans: inhibitory components of the glial scar. Prog. Brain Res. 132, 611-619. doi: 10.1016/s0079-6123(01)32106-4

Bai, Y., Zhu, X., Chao, J., Zhang, Y., Qian, C., Li, P., et al. (2015). Pericytes contribute to the disruption of the cerebral endothelial barrier via increasing VEGF expression: implications for stroke. PLoS One 10:e0124362. doi: 10.1371/ journal.pone.0124362

Balabanov, R., Washington, R., Wagnerova, J., and Dore-Duffy, P. (1996). CNS microvascular pericytes express macrophage-like function, cell surface integrin alpha M, and macrophage marker ED-2. Microvasc. Res. 52, 127-142. doi: 10.1006/mvre.1996.0049

Bell, R. D., Winkler, E. A., Sagare, A. P., Singh, I., LaRue, B., Deane, R., et al. (2010). Pericytes control key neurovascular functions and neuronal phenotype in the adult brain and during brain aging. Neuron 68, 409-427. doi: 10.1016/j.neuron. 2010.09.043

Bell, R. D., Winkler, E. A., Singh, I., Sagare, A. P., Deane, R., Wu, Z., et al. (2012). Apolipoprotein E controls cerebrovascular integrity via cyclophilin A. Nature 485, 512-516. doi: 10.1038/nature11087

Berger, M., Bergers, G., Arnold, B., Hämmerling, G. J., and Ganss, R. (2005). Regulator of G-protein signaling-5 induction in pericytes coincides with active vessel remodeling during neovascularization. Blood 105, 1094-1101. doi: 10 . 1182/blood-2004-06-2315

Birbrair, A., Zhang, T., Files, D. C., Mannava, S., Smith, T., Wang, Z. M., et al. (2014). Type-1 pericytes accumulate after tissue injury and produce collagen in an organ-dependent manner. Stem Cell Res. Ther. 5:122. doi: 10.1186/scrt512 isotype-specific biomarkers, as well as drugs targeting pericytes or modulating their activities and autotransplantation of pericytes. Acquiring more information about pericytes will help us in studying pericytes in a novel way and facilitating the development of therapies for ischemic stroke.

\section{AUTHOR CONTRIBUTIONS}

LC searched for relevant literature and drafted the manuscript. YZ and MC searched for relevant literature and revised the manuscript critically. LL and WZ provided professional guidance for this review and performed a final check of the manuscript. All authors contributed to the review of this manuscript and approved the submitted version.

\section{FUNDING}

This study was supported by the National Natural Science Foundation of China 81870926 to LL.

Birbrair, A., Zhang, T., Wang, Z. M., Messi, M. L., Enikolopov, G. N., Mintz, A., et al. (2013a). Role of pericytes in skeletal muscle regeneration and fat accumulation. Stem Cells Dev. 22, 2298-2314. doi: 10.1089/scd.2012.0647

Birbrair, A., Zhang, T., Wang, Z. M., Messi, M. L., Enikolopov, G. N., Mintz, A., et al. (2013b). Skeletal muscle pericyte subtypes differ in their differentiation potential. Stem Cell Res. 10, 67-84. doi: 10.1016/j.scr.2012.09.003

Bondjers, C., Kalén, M., Hellström, M., Scheidl, S. J., Abramsson, A., Renner, O., et al. (2003). Transcription profiling of platelet-derived growth factor-Bdeficient mouse embryos identifies RGS5 as a novel marker for pericytes and vascular smooth muscle cells. Am. J. Pathol. 162, 721-729. doi: 10.1016/s00029440(10)63868-0

Boya, J. (1976). An ultrastructural study of the relationship between pericytes and cerebral macrophages. Acta Anat. 95, 598-608. doi: 10.1159/000144648

Cai, C., Fordsmann, J. C., Jensen, S. H., Gesslein, B., Lønstrup, M., Hald, B. O., et al. (2018). Stimulation-induced increases in cerebral blood flow and local capillary vasoconstriction depend on conducted vascular responses. Proc. Natl. Acad. Sci. U.S.A. 115, E5796-E5804.

Castejon, O. J. (1984). Submicroscopic changes of cortical capillary pericytes in human perifocal brain edema. J. Submicrosc. Cytol. 16, 601-608.

Chen, Y. T., Chang, F. C., Wu, C. F., Chou, Y. H., Hsu, H. L., Chiang, W. C., et al. (2011). Platelet-derived growth factor receptor signaling activates pericytemyofibroblast transition in obstructive and post-ischemic kidney fibrosis. Kidney Int. 80, 1170-1181. doi: 10.1038/ki.2011.208

Cheng, R., and Ma, J. X. (2015). Angiogenesis in diabetes and obesity. Rev. Endocr. Metab. Disord. 16, 67-75.

Cho, H., Kozasa, T., Bondjers, C., Betsholtz, C., and Kehrl, J. H. (2003). Pericytespecific expression of Rgs5: implications for PDGF and EDG receptor signaling during vascular maturation. FASEB J. 17, 440-442.

Cregg, J. M., DePaul, M. A., Filous, A. R., Lang, B. T., Tran, A., and Silver, J. (2014). Functional regeneration beyond the glial scar. Exp. Neurol. 253, 197-207. doi: 10.1016/j.expneurol.2013.12.024

Dalkara, T. (2019). Pericytes: a novel target to improve success of recanalization therapies. Stroke 50, 2985-2991. doi: 10.1161/strokeaha.118.023590

Daneman, R., and Prat, A. (2015). The blood-brain barrier. Cold Spring Harb. Perspect. Biol. 7:a020412.

Daneman, R., Zhou, L., Kebede, A. A., and Barres, B. A. (2010). Pericytes are required for blood-brain barrier integrity during embryogenesis. Nature 468, 562-566. doi: 10.1038/nature09513

Deguchi, K., Liu, N., Liu, W., Omote, Y., Kono, S., Yunoki, T., et al. (2014). Pericyte protection by edaravone after tissue plasminogen activator treatment in rat cerebral ischemia. J. Neurosci. Res. 92, 1509-1519. doi: 10.1002/jnr.23420 
Dohgu, S., and Banks, W. A. (2013). Brain pericytes increase the lipopolysaccharide-enhanced transcytosis of HIV-1 free virus across the in vitro blood-brain barrier: evidence for cytokine-mediated pericyte-endothelial cell crosstalk. Fluids Barriers CNS 10:23. doi: 10.1186/2045-8118-10-23

Dohgu, S., Takata, F., Yamauchi, A., Nakagawa, S., Egawa, T., Naito, M., et al. (2005). Brain pericytes contribute to the induction and up-regulation of bloodbrain barrier functions through transforming growth factor-beta production. Brain Res. 1038, 208-215. doi: 10.1016/j.brainres.2005.01.027

Dore-Duffy, P., and LaManna, J. C. (2007). Physiologic angiodynamics in the brain. Antioxid Redox Signal. 9, 1363-1371. doi: 10.1089/ars.2007.1713

Duan, L., Zhang, X. D., Miao, W. Y., Sun, Y. J., Xiong, G., Wu, Q., et al. (2018). PDGFR $\beta$ Cells rapidly relay inflammatory signal from the circulatory system to neurons via chemokine CCL2. Neuron 100, 183.e8-200.e8.

Etchevers, H. C., Vincent, C., Le Douarin, N. M., and Couly, G. F. (2001). The cephalic neural crest provides pericytes and smooth muscle cells to all blood vessels of the face and forebrain. Development 128, 1059-1068. doi: 10.1242/ dev.128.7.1059

Faal, T., Phan, D. T. T., Davtyan, H., Scarfone, V. M., Varady, E., Blurton-Jones, M., et al. (2019). Induction of mesoderm and neural crest-derived pericytes from human pluripotent stem cells to study blood-brain barrier interactions. Stem Cell Rep. 12, 451-460. doi: 10.1016/j.stemcr.2019.01.005

Fawcett, J. W., and Asher, R. A. (1999). The glial scar and central nervous system repair. Brain Res. Bull. 49, 377-391. doi: 10.1016/s0361-9230(99)00072-6

Fernández-Klett, F., Offenhauser, N., Dirnagl, U., Priller, J., and Lindauer, U. (2010). Pericytes in capillaries are contractile in vivo, but arterioles mediate functional hyperemia in the mouse brain. Proc. Natl. Acad. Sci. U.S.A. 107, 22290-22295. doi: 10.1073/pnas. 1011321108

Fitch, M. T., and Silver, J. (2008). CNS injury, glial scars, and inflammation: inhibitory extracellular matrices and regeneration failure. Exp. Neurol. 209, 294-301. doi: 10.1016/j.expneurol.2007.05.014

Gautam, J., and Yao, Y. (2018). Roles of pericytes in stroke pathogenesis. Cell Transplant 27, 1798-1808. doi: 10.1177/0963689718768455

Geranmayeh, M. H., Rahbarghazi, R., and Farhoudi, M. (2019). Targeting pericytes for neurovascular regeneration. Cell Commun. Signal. 17:26.

Gouveia, A., Seegobin, M., Kannangara, T. S., He, L., Wondisford, F., Comin, C. H., et al. (2017). The aPKC-CBP pathway regulates post-stroke neurovascular remodeling and functional recovery. Stem Cell Rep. 9, 1735-1744. doi: 10.1016/ j.stemcr.2017.10.021

Hagedorn, M., Balke, M., Schmidt, A., Bloch, W., Kurz, H., Javerzat, S., et al. (2004). VEGF coordinates interaction of pericytes and endothelial cells during vasculogenesis and experimental angiogenesis. Dev. Dyn. 230, 23-33. doi: 10.1002/dvdy. 20020

Hall, C. N., Reynell, C., Gesslein, B., Hamilton, N. B., Mishra, A., Sutherland, B. A., et al. (2014). Capillary pericytes regulate cerebral blood flow in health and disease. Nature 508, 55-60. doi: 10.1038/nature 13165

Hartmann, D. A., Berthiaume, A. A., Grant, R. I., Harrill, S. A., Koski, T., Tieu, T., et al. (2021). Brain capillary pericytes exert a substantial but slow influence on blood flow. Nat. Neurosci. 24, 633-645. doi: 10.1038/s41593-020-00793-2

Hartmann, D. A., Underly, R. G., Grant, R. I., Watson, A. N., Lindner, V., and Shih, A. Y. (2015). Pericyte structure and distribution in the cerebral cortex revealed by high-resolution imaging of transgenic mice. Neurophotonics 2:041402. doi: 10.1117/1.nph.2.4.041402

Hill, R. A., Tong, L., Yuan, P., Murikinati, S., Gupta, S., and Grutzendler, J. (2015). Regional blood flow in the normal and ischemic brain is controlled by arteriolar smooth muscle cell contractility and not by capillary pericytes. Neuron 87, 95-110. doi: 10.1016/j.neuron.2015.06.001

Höke, A., and Silver, J. (1996). Proteoglycans and other repulsive molecules in glial boundaries during development and regeneration of the nervous system. Prog. Brain Res. 108, 149-163. doi: 10.1016/s0079-6123(08)62538-8

Hori, S., Ohtsuki, S., Hosoya, K., Nakashima, E., and Terasaki, T. (2004). A pericyte-derived angiopoietin-1 multimeric complex induces occludin gene expression in brain capillary endothelial cells through Tie-2 activation in vitro. J. Neurochem. 89, 503-513. doi: 10.1111/j.1471-4159.2004.02343.x

Janghorbani, M., Hu, F. B., Willett, W. C., Li, T. Y., Manson, J. E., Logroscino, G., et al. (2007). Prospective study of type 1 and type 2 diabetes and risk of stroke subtypes: the Nurses'. Health Study Diabetes Care 30, 1730-1735. doi: $10.2337 / \mathrm{dc} 06-2363$
Kamouchi, M., Kitazono, T., Ago, T., Wakisaka, M., Ooboshi, H., Ibayashi, S., et al. (2004). Calcium influx pathways in rat CNS pericytes. Brain Res. Mol. Brain Res. 126, 114-120. doi: 10.1016/j.molbrainres.2004.03.008

Kawano, H., Kimura-Kuroda, J., Komuta, Y., Yoshioka, N., Li, H. P., Kawamura, K., et al. (2012). Role of the lesion scar in the response to damage and repair of the central nervous system. Cell Tissue Res. 349, 169-180. doi: 10.1007/s00441012-1336-5

Khennouf, L., Gesslein, B., Brazhe, A., Octeau, J. C., Kutuzov, N., Khakh, B. S., et al. (2018). Active role of capillary pericytes during stimulation-induced activity and spreading depolarization. Brain 141, 2032-2046. doi: 10.1093/ brain/awy143

Kisler, K., Nelson, A. R., Montagne, A., and Zlokovic, B. V. (2017). Cerebral blood flow regulation and neurovascular dysfunction in Alzheimer disease. Nat. Rev. Neurosci. 18, 419-434. doi: 10.1038/nrn.2017.48

Kisler, K., Nikolakopoulou, A. M., Sweeney, M. D., Lazic, D., Zhao, Z., and Zlokovic, B. V. (2020). Acute ablation of cortical pericytes leads to rapid neurovascular uncoupling. Front. Cell Neurosci. 14:27. doi: 10.3389/fncel.2020. 00027

Kofler, N. M., Cuervo, H., Uh, M. K., Murtomäki, A., and Kitajewski, J. (2015). Combined deficiency of Notch1 and Notch3 causes pericyte dysfunction, models CADASIL, and results in arteriovenous malformations. Sci. Rep. 5:16449.

Korn, J., Christ, B., and Kurz, H. (2002). Neuroectodermal origin of brain pericytes and vascular smooth muscle cells. J. Comp. Neurol. 442, 78-88. doi: 10.1002/ cne. 1423

Kovac, A., Erickson, M. A., and Banks, W. A. (2011). Brain microvascular pericytes are immunoactive in culture: cytokine, chemokine, nitric oxide, and LRP-1 expression in response to lipopolysaccharide. J. Neuroinflammation 8:139. doi: 10.1186/1742-2094-8-139

Kurz, H. (2009). Cell lineages and early patterns of embryonic CNS vascularization. Cell Adh. Migr. 3, 205-210. doi: 10.4161/cam.3.2.7855

Le Beux, Y. J., and Willemot, J. (1978). Actin- and myosin-like filaments in rat brain pericytes. Anat. Rec. 190, 811-826. doi: 10.1002/ar.1091900404

Li, H. P., Komuta, Y., Kimura-Kuroda, J., van Kuppevelt, T. H., and Kawano, H. (2013). Roles of chondroitin sulfate and dermatan sulfate in the formation of a lesion scar and axonal regeneration after traumatic injury of the mouse brain. J. Neurotrauma 30, 413-425. doi: 10.1089/neu.2012.2513

Li, Q., Khatibi, N., and Zhang, J. H. (2014). Vascular neural network: the importance of vein drainage in stroke. Transl. Stroke Rese. 5, 163-166. doi: 10.1007/s12975-014-0335-0

Makihara, N., Arimura, K., Ago, T., Tachibana, M., Nishimura, A., Nakamura, K., et al. (2015). Involvement of platelet-derived growth factor receptor $\beta$ in fibrosis through extracellular matrix protein production after ischemic stroke. Exp. Neurol. 264, 127-134. doi: 10.1016/j.expneurol.2014.12.007

McKeon, R. J., Jurynec, M. J., and Buck, C. R. (1999). The chondroitin sulfate proteoglycans neurocan and phosphacan are expressed by reactive astrocytes in the chronic CNS glial scar. J. Neurosci. 19, 10778-10788. doi: 10.1523/jneurosci. 19-24-10778.1999

Moskowitz, M. A., Lo, E. H., and Iadecola, C. (2010). The science of stroke: mechanisms in search of treatments. Neuron 67, 181-198. doi: 10.1016/j. neuron.2010.07.002

Nakagomi, T., Kubo, S., Nakano-Doi, A., Sakuma, R., Lu, S., Narita, A., et al. (2015). Brain vascular pericytes following ischemia have multipotential stem cell activity to differentiate into neural and vascular lineage cells. Stem Cells 33, 1962-1974. doi: 10.1002/stem.1977

Nelson, A. R., Sagare, M. A., Wang, Y., Kisler, K., Zhao, Z., and Zlokovic, B. V. (2020). Channelrhodopsin excitation contracts brain pericytes and reduces blood flow in the aging mouse brain in vivo. Front. Aging Neurosci. 12:108. doi: 10.3389/fnagi.2020.00108

Neuhaus, A. A., Couch, Y., Sutherland, B. A., and Buchan, A. M. (2017). Novel method to study pericyte contractility and responses to ischaemia in vitro using electrical impedance. J. Cereb. Blood Flow Metab. 37, 2013-2024. doi: $10.1177 / 0271678 \times 16659495$

Nikolakopoulou, A. M., Montagne, A., Kisler, K., Dai, Z., Wang, Y., Huuskonen, M. T., et al. (2019). Pericyte loss leads to circulatory failure and pleiotrophin depletion causing neuron loss. Nat. Neurosci. 22, 1089-1098. doi: 10.1038/ s41593-019-0434-z 
Nishimura, A., Ago, T., Kuroda, J., Arimura, K., Tachibana, M., Nakamura, K., et al. (2016). Detrimental role of pericyte Nox4 in the acute phase of brain ischemia. J. Cereb. Blood Flow Metab. 36, 1143-1154. doi: 10.1177/0271678x15606456

Özen, I., Deierborg, T., Miharada, K., Padel, T., Englund, E., Genové, G., et al. (2014). Brain pericytes acquire a microglial phenotype after stroke. Acta Neuropathol. 128, 381-396. doi: 10.1007/s00401-014-1295-x

Ozen, I., Roth, M., Barbariga, M., Gaceb, A., Deierborg, T., Genove, G., et al. (2018). Loss of regulator of G-protein signaling 5 leads to neurovascular protection in stroke. Stroke 49, 2182-2190. doi: 10.1161/strokeaha.118.020124

Persidsky, Y., Ramirez, S. H., Haorah, J., and Kanmogne, G. D. (2006). Blood-brain barrier: structural components and function under physiologic and pathologic conditions. J. Neuroimmune Pharmacol. 1, 223-236. doi: 10.1007/s11481-0069025-3

Ramsauer, M., Krause, D., and Dermietzel, R. (2002). Angiogenesis of the bloodbrain barrier in vitro and the function of cerebral pericytes. FASEB J. 16, 1274-1276. doi: 10.1096/fj.01-0814fje

Rouget, C. M. (1873). Memoire sur le developpement, de la structure et les proprietes physiologiques des capillaires sanguins et lympha-tiques. Arch. Physiol. Norm. Path. 5, 603-663.

Rungta, R. L., Chaigneau, E., Osmanski, B. F., and Charpak, S. (2018). Vascular compartmentalization of functional hyperemia from the synapse to the Pia. Neuron 99, 362.e4-375.e4.

Sakuma, R., Kawahara, M., Nakano-Doi, A., Takahashi, A., Tanaka, Y., Narita, A., et al. (2016). Brain pericytes serve as microglia-generating multipotent vascular stem cells following ischemic stroke. J. Neuroinflammation 13:57.

Shen, W., Li, S., Chung, S. H., Zhu, L., Stayt, J., Su, T., et al. (2011). Tyrosine phosphorylation of VE-cadherin and claudin-5 is associated with TGF- $\beta 1$ induced permeability of centrally derived vascular endothelium. Eur. J Cell Biol. 90, 323-332. doi: 10.1016/j.ejcb.2010.10.013

Shibahara, T., Ago, T., Nakamura, K., Tachibana, M., Yoshikawa, Y., Komori, M., et al. (2020). Pericyte-mediated tissue repair through PDGFR $\beta$ promotes peri-infarct astrogliosis, oligodendrogenesis, and functional recovery after acute ischemic stroke. eNeuro 7:ENEURO.0474-19.2020.

Shimotake, J., Derugin, N., Wendland, M., Vexler, Z. S., and Ferriero, D. M. (2010). Vascular endothelial growth factor receptor-2 inhibition promotes cell death and limits endothelial cell proliferation in a neonatal rodent model of stroke. Stroke 41, 343-349. doi: 10.1161/strokeaha.109.564229

Sieczkiewicz, G. J., and Herman, I. M. (2003). TGF-beta 1 signaling controls retinal pericyte contractile protein expression. Microvasc. Res. 66, 190-196. doi: 10.1016/s0026-2862(03)00055-4

Silver, J., and Miller, J. H. (2004). Regeneration beyond the glial scar. Nat. Rev. Neurosci. 5, 146-156. doi: 10.1038/nrn1326

Sinha, S., Hoofnagle, M. H., Kingston, P. A., McCanna, M. E., and Owens, G. K. (2004). Transforming growth factor-betal signaling contributes to development of smooth muscle cells from embryonic stem cells. Am. J. Physiol. Cell Physiol. 287, C1560-C1568.

Stebbins, M. J., Gastfriend, B. D., Canfield, S. G., Lee, M.-S., Richards, D., Faubion, M. G., et al. (2019). Human pluripotent stem cell-derived brain pericyte-like cells induce blood-brain barrier properties. Sci. Adv. 5:eaau7375. doi: 10.1126/ sciadv.aau7375

Stenzel, D., Nye, E., Nisancioglu, M., Adams, R. H., Yamaguchi, Y., Gerhardt, H., et al. (2009). Peripheral mural cell recruitment requires cell-autonomous heparan sulfate. Blood 114, 915-924. doi: 10.1182/blood-2008-10-18 6239

Sun, J., Huang, Y., Gong, J., Wang, J., Fan, Y., Cai, J., et al. (2020). Transplantation of hPSC-derived pericyte-like cells promotes functional recovery in ischemic stroke mice. Nat. Commun. 11:5196.

Sun, M., Chen, X., Yin, Y. X., Gao, Y., Zhang, L., Chen, B., et al. (2020). Role of pericyte-derived SENP1 in neuronal injury after brain ischemia. CNS Neurosci. Ther. 26, 815-828. doi: 10.1111/cns.13398

Terasaki, Y., Liu, Y., Hayakawa, K., Pham, L. D., Lo, E. H., Ji, X., et al. (2014). Mechanisms of neurovascular dysfunction in acute ischemic brain. Curr. Med. Chem. 21, 2035-2042. doi: 10.2174/0929867321666131228223400

Tom, V. J., Doller, C. M., Malouf, A. T., and Silver, J. (2004). Astrocyte-associated fibronectin is critical for axonal regeneration in adult white matter. J. Neurosci. 24, 9282-9290. doi: 10.1523/jneurosci.2120-04.2004

van Bruggen, N., Thibodeaux, H., Palmer, J. T., Lee, W. P., Fu, L., Cairns, B., et al. (1999). VEGF antagonism reduces edema formation and tissue damage after ischemia/reperfusion injury in the mouse brain. J. Clin. Invest. 104, 1613-1620. doi: $10.1172 /$ jci8218

Weis, S. M., and Cheresh, D. A. (2005). Pathophysiological consequences of VEGFinduced vascular permeability. Nature 437, 497-504. doi: 10.1038/nature03987

Yanqing, Z., Yu-Min, L., Jian, Q., Bao-Guo, X., and Chuan-Zhen, L. (2006). Fibronectin and neuroprotective effect of granulocyte colony-stimulating factor in focal cerebral ischemia. Brain Res. 1098, 161-169. doi: 10.1016/j.brainres. 2006.02.140

Yemisci, M., Gursoy-Ozdemir, Y., Vural, A., Can, A., Topalkara, K., and Dalkara, T. (2009). Pericyte contraction induced by oxidative-nitrative stress impairs capillary reflow despite successful opening of an occluded cerebral artery. Nat. Med. 15, 1031-1037. doi: 10.1038/nm.2022

Zechariah, A., ElAli, A., Doeppner, T. R., Jin, F., Hasan, M. R., Helfrich, I., et al. (2013). Vascular endothelial growth factor promotes pericyte coverage of brain capillaries, improves cerebral blood flow during subsequent focal cerebral ischemia, and preserves the metabolic penumbra. Stroke 44, 1690-1697. doi: 10.1161/strokeaha.111.000240

Zhang, Z. G., Zhang, L., Jiang, Q., Zhang, R., Davies, K., Powers, C., et al. (2000). VEGF enhances angiogenesis and promotes blood-brain barrier leakage in the ischemic brain. J. Clin. Invest. 106, 829-838. doi: 10.1172/jci9369

Zimmermann, K. W. (1923). Der feinere bau der blutcapillaren. Z. Anat. Entwicklungsgesch. 68:29109.

Zlokovic, B. V. (2013). Cerebrovascular effects of apolipoprotein E: implications for Alzheimer disease. JAMA Neurol. 70, 440-444. doi: 10.1001/jamaneurol.2013. 2152

Conflict of Interest: The authors declare that the research was conducted in the absence of any commercial or financial relationships that could be construed as a potential conflict of interest.

Copyright (c) 2021 Cao, Zhou, Chen, Li and Zhang. This is an open-access article distributed under the terms of the Creative Commons Attribution License (CC BY). The use, distribution or reproduction in other forums is permitted, provided the original author(s) and the copyright owner(s) are credited and that the original publication in this journal is cited, in accordance with accepted academic practice. No use, distribution or reproduction is permitted which does not comply with these terms. 